\title{
Predição de Características Quantitativas de Carcaças de Suínos pela Técnica de Ultra-Sonografia em Tempo Real ${ }^{1}$
}

\section{Wilson Moreira Dutra Jr.2 ${ }^{2}$, Aloízio Soares Ferreira ${ }^{3}$, Jaime Urdapilleta Tarouco ${ }^{4}$, Juarez Lopes Donzele ${ }^{3}$, Ricardo Frederico Euclydes ${ }^{3}$, Luiz Fernando Teixeira Albino ${ }^{3}$, Leandro Lunardini Cardoso ${ }^{4}$, Simone dos Passos Fernandes ${ }^{4}$}

RESUMO - Foram utilizadas 48 fêmeas suínas, para se determinar o rendimento de cortes comerciais, após abate e dissecação das carcaças dos animais aos 50, 60, 70, 80, 90, 100, 110 e $120 \mathrm{~kg}$. Foram tomadas medidas ultra-sônicas in vivo para área de olho de lombo e espessura de toucinho. Os preditores utilizados para estimar o rendimento de carne magra foram espessura de toucinho e área de olho de lombo, além das medidas feitas nos diferentes cortes, como peso do corte do pernil, peso do músculo do pernil, peso do músculo da paleta, peso do músculo do carré, peso do músculo da copa, e assim por diante, além dos dados de peso de músculo da carcaça total, peso da gordura da carcaça total, peso de ossos da carcaça total e peso de pele da carcaça total. Foram desenvolvidas equações de predição por meio de regressão linear simples e múltipla, a partir dos dados da dissecação e das medidas in vivo da área de olho de lombo, feitas por ultra-sonografia em tempo real. As equações de predição para quantificar os principais componentes dos cortes suínos tiveram ótimo ajuste, com $R^{2}$ superiores a 0,84 para músculos e desviopadrão residual de 0,62 a 0,94. A técnica de ultra-sonografia em tempo real apresenta alta correlação com as medidas tomadas nas carcaças dos suínos e, por isso, estima a quantidade de carne na carcaça e o rendimento dos cortes comerciais com grande precisão. Concluiu-se que a técnica de ultra-sonografia em tempo real é acurada para predizer as principais características das carcaças dos suínos medidas in vivo.

Palavras-chave: área de olho de lombo, cortes comerciais, espessura de toucinho, tempo real, ultra-sonografia

\section{Prediction of Quantitative Swine Carcass Traits by the Real-Time Ultrasonicgraphy Technology}

\begin{abstract}
Forty-eight female pigs were used to determine the commercial cut yields after slaughter of the animals at 50, 60, 70, 80, $90,100,110$ and $120 \mathrm{~kg}$ and dissection of the carcasses. Life ultrasonic measures were taken to measure the longissimus muscle area and for backfat thickness. The predictors used to estimate the lean meat yield were backfat thickness and longissimus muscle area. Besides the measures made in the different cuts, as ham cut weight, ham muscle weight, palette muscle weight, and carré muscle weight and pantry muscle weight. Additionally, the data of the total carcass muscle weight, total carcass fat weight, total carcass bone weight and total carcass skin weight, were also used. Prediction equations were developed using simple and multiple linear regressions using the logistic model from the dissection data and from the live ultrasonic measure data. Prediction equations were developed through out simple and multiple linear regressions using a logistic model from the dissection and live measures made by real-time ultrasound technology data. The prediction equation to determine the amounts the main components of the meat pork cuts presented a great adjustment, with $\mathrm{R}^{2}$ superiors to 0.84 for muscles, with residual standard deviation (RSD) 0.62 to 0.94 . The real-time ultrasonograph technology presented high correlation with the measures taking in the pork carcasses and could be used to estimate the amount of lean meat in the pork carcasses and also to estimate the commercial pork cut yields with great precision. It was concluded that the real-time ultrasonograph technology is accurate to predict the main pork carcass traits measured in the live animal.
\end{abstract}

Key Words: backfat thickness, commercial cuts, loin eye area, real-time, ultrasonicgraphy

\section{Introdução}

Aumentar a quantidade e melhorar a qualidade da carne na carcaça de suínos tem sido o objetivo não somente da indústria, como também do produtor de suínos, pois melhora a rentabilidade e diminui os custos de produção. Dessa forma, assume papel importante na cadeia produtiva de carnes e derivados o conhecimento das quantidades de tecidos no animal, antes mesmo do abate, uma vez que as mudanças de peso corporal dos animais podem ser atribuídas a alguns tecidos específicos, como, por exemplo, a gordura, que, dependendo da quantidade na carcaça, pode ser indesejável. Estudos para predizer a quantidade de tecidos nas carcaças têm sido feitos por vários pesquisadores, a partir dos quais têm-se

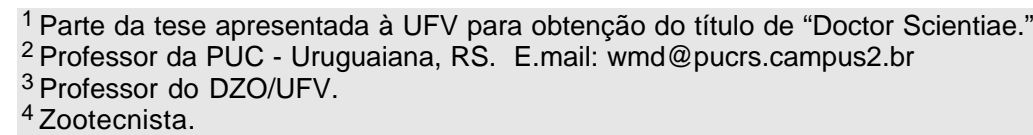


gerado equações de regressão, que, têm tido aplicação restrita para determinados grupos genéticos.

Existem várias técnicas para avaliação e classificação de carcaças de suínos, destacando-se as técnicas com uso de aparelhos ópticos, durante o processo de abate, e as técnicas com uso de ultrasonografia, que pode ser usada tanto durante o processo de abate, quanto no animal in vivo. Porém, as técnicas mais utilizadas no mundo têm sido aquelas com uso de aparelhos ópticos (IRGANG et al., 1998), com medições da profundidade de gordura e músculo em determinados pontos da carcaça, durante o processo de abate dos suínos.

Em pesquisas recentes, tem sido verificado que os aparelhos ópticos apresentam acuracidade inferior aos aparelhos de ultra-sonografia, principalmente em termos de quantidade de músculo (JOYAL et al., 1987) e, também, a avaliação de carcaças de suínos pela técnica de ultra-sonografia em tempo real tem sido precisa, apresentando alta correlação (85 a 97\%) entre os valores observados e as medidas obtidas diretamente nas carcaças (SCHINCKEL et al., 1994). Se estas observações forem confirmadas, é possível que o uso desta técnica possa permitir avaliação dos animais in vivo e, conseqüentemente, se tornar viável em nível de granja, possibilitando, dessa forma, que os produtores selecionem os animais de melhores carcaças com maior eficiência, rapidez e precisão (LO et al., 1992 a, b; LIU e STOUFFER, 1995; e SATHER et al., 1996).

Contudo, têm-se verificado resultados discrepantes com relação à predição da qualidade da carne por meio das análises de imagens ultra-sônicas em tempo real, em especial com relação à medição da gordura intramuscular, ou seja, do marmoreio (WILSON, 1996).

Assim, constatou-se a necessidade de testar se a técnica de ultra-sonografia em tempo real é acurada para predizer as principais características de carcaça dos suínos in vivo e se a partir delas é possível estimar a quantidade de carne na carcaça e o rendimento de cortes comerciais com precisão.

\section{Material e Métodos}

Foram selecionadas 48 fêmeas suínas de um grupo de 500, da linhagem Camborough 22, com peso médio inicial de $25,53 \mathrm{~kg}$ e idade média de 62 dias para uso no experimento.

Para a realização das medidas ultra-sônicas, utilizou-se um conjunto de equipamentos constituído de: uma ecocâmera ALOKA SSD 500, uma probe de $11,5 \mathrm{~cm}$ e 3,5 MHz, duas guias acústicas ou standoff, para medidas longitudinais e transversais, um software AUSKey System V-5.0, for windows, acoplado a um notebook, com cartão PCMCIA.

As medidas ultra-sônicas foram coletadas após a contenção dos animais, utilizando-se primeiramente a guia transversal para tomada da área de olho de lombo e, em seguida, uma medida longitudinal para a espessura de toucinho e profundidade de músculo. A primeira medida foi coletada transversalmente entre a décima e a décima primeira costela, por meio de uma guia acústica de polivinil (Figura 1), enquanto a segunda medida foi tomada com uma guia acústica reta, na área compreendida entre a décima e a última costela dos suínos, a $5 \mathrm{~cm}$ abaixo da linha média (Figura 2).

Para o cálculo do rendimento de carne magra, área de olho de lombo e espessura de toucinho, utilizou-se o "software" AUSKey System V-5.0, for windows, desenvolvido por LIU (1997), o qual apresenta equações de predição que consideram o peso do animal, a área de olho de lombo estimada e a espessura de toucinho, como variáveis.

Os animais foram abatidos no frigorífico da COOPERJACUÍ, Central de Cooperativas do Alto

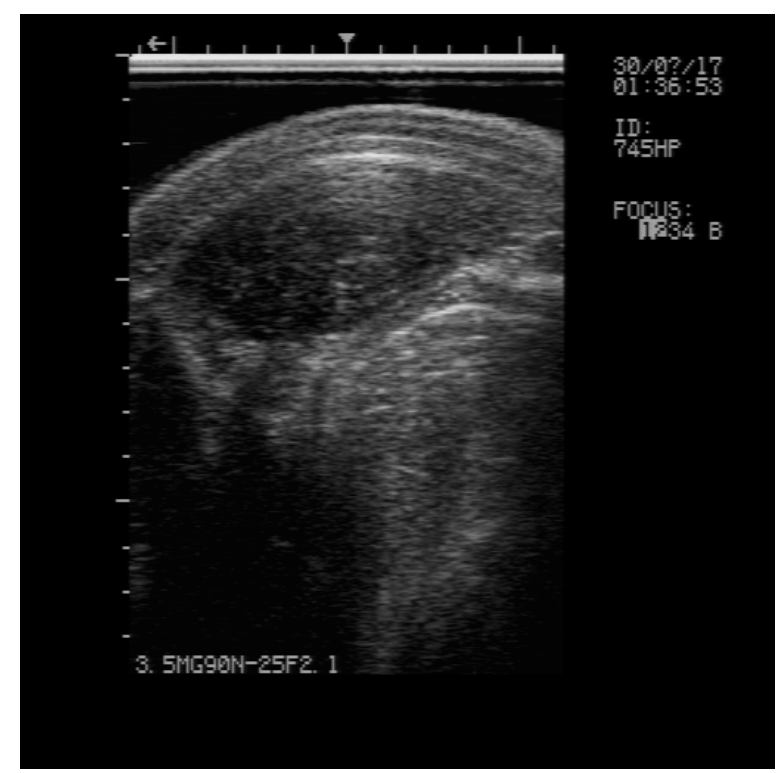

Figura 1 - Imagem transversal do músculo Longissimus dorsi, entre a décima e a décima primeira costela, em suínos.

Figure 1 - Transversal image of Longissimus dorsi muscle from the $10^{\text {th }}$ ans $11^{\text {th }}$ rib, in swines. 


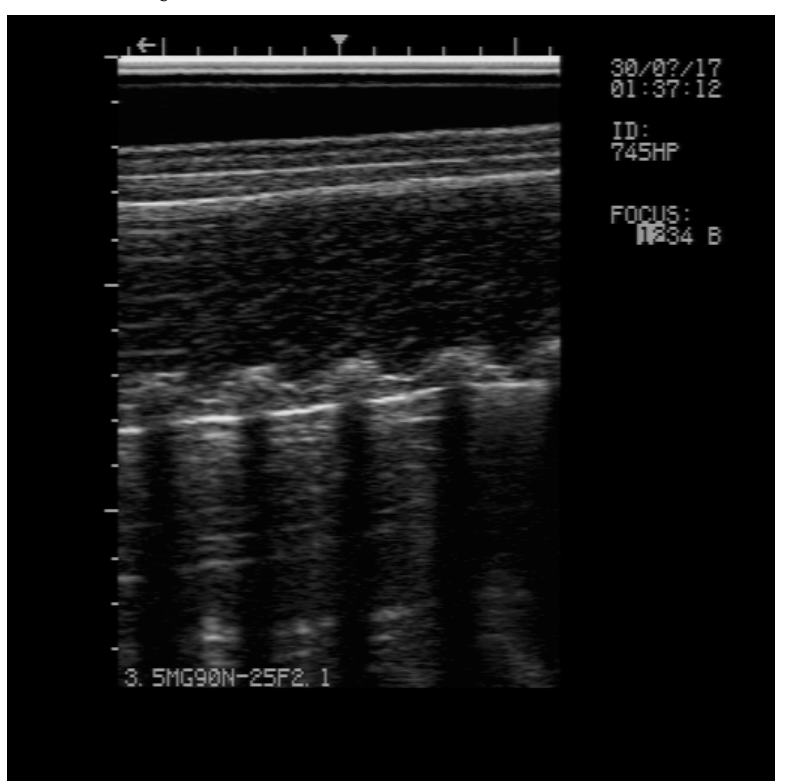

Figura 2 - Imagem longitudinal entre a décima e a última costela, em suínos.

Figure 2 - Longitudinal image from the $10^{\text {th }}$ and $11^{\text {th }}$ rib, in swines.

Jacuí, onde foram dissecadas, também, suas carcaças.

Foram realizadas medições a $5 \mathrm{~cm}$ abaixo da linha média, na altura da décima costela na carcaça quente dos suínos abatidos, para estimar espessura de toucinho (ET) e profundidade de músculo (PM), por aparelho óptico Hennessy, de acordo com o procedimento adotado pela indústria de carnes de suínos no Brasil.

Os cálculos de porcentagem de carne magra, porcentagem de gordura e porcentagem de ossos foram realizados adotando-se a seguinte fórmula:

$$
\mathrm{R}=\mathrm{P} * \mathrm{PQF} / \mathrm{PCQ}_{\text {Limp }} * 100
$$

em que: $\mathrm{R}=$ rendimento de carne magra, gordura, ou ossos, conforme o caso; $\mathrm{P}=$ peso total de músculo, gordura ou ossos, conforme o caso; $\mathrm{PQF}=$ porcentagem de quebra de frigorificação; $\mathrm{PCQ}_{\text {Limp }}=$ peso da carcaça quente limpa.

Para o cálculo do peso do músculo, adotou-se a soma das porções de músculo da metade esquerda multiplicada por dois, uma vez que a diferença de peso entre uma metade e outra ficou abaixo de $1 \%$ na média.

A predição de características quantitativas de carcaças de suínos, por meio da técnica de ultrasonografia em tempo real, foi feita utilizando-se os dados da dissecação das carcaças, dos diferentes cortes comerciais em correlações, com a área de olho de lombo medida por ultra-som.
Os preditores utilizados para estimar o rendimento de carne magra foram a espessura de toucinho e a área de olho de lombo medidas na carcaça, a área de olho de lombo medida por ultra-som, além das medidas feitas nos diferentes cortes, como peso do corte do pernil, peso do músculo do pernil, peso do músculo da paleta, peso do músculo do carré, peso do músculo da copa, e assim por diante, além dos dados de peso de músculo da carcaça total, peso da gordura da carcaça total, peso de ossos da carcaça total e peso de pele da carcaça total.

A variável carne foi definida como o peso do filé e do lombo, despojados da gordura, mais a quantidade de carne extraída do pernil, da barriga, das costelas, do carré, da copa e da paleta, despojadas da respectiva gordura. A variável gordura foi definida como o peso da gordura extraída de papada, paleta, copa, carré, barriga e costelas e pernil; a variável ossos, como o somatório da quantidade de ossos extraídos de pernil, paleta, copa, carré e costelas; e a variável pele, como o somatório da quantidade de pele extraída da superfície de pernil, paleta, carré e barriga.

Foram desenvolvidas equações de predição lineares simples e múltiplas, a partir dos dados da dissecação e das medidas da área de olho de lombo feitas in vivo, pela técnica de ultra-sonografia em tempo real, e das medidas verificadas com o aparelho óptico e nas carcaças resfriadas, utilizando-se o Sistema de Análises Estatísticas e Genéticas SAEG (UNIVERSIDADE FEDERAL DE VIÇOSA - UFV, 1998).

\section{Resultados e Discussão}

Os resultados relativos às equações de predição de cortes cárneos de carcaça de suínos, por meio da área de olho de lombo medida por ultra-sonografia em tempo real (AOLUS), podem ser visualizados na Tabela 1.

Os valores de $\mathrm{R}^{2}$ obtidos nesta pesquisa foram superiores aos obtidos por GRESHAM et al. (1994), que verificaram valores de $\mathrm{R}^{2}$ da ordem de $0,76 \mathrm{e}$ 0,81 , para estimativas de cortes cárneos (pernil e paleta), por meio de avaliações ultra-sônicas realizadas em animais vivos e nas carcaças dos mesmos, respectivamente. Os mesmos autores verificaram, também, valores de desvio-padrão residual (DPR) de 2,77 e 2,48 para os mesmos cortes. Assim, constata-se que os resultados obtidos neste estudo foram mais consistentes que os encontrados em estudos similares.

Quanto às estimativas para se medir a composição em tecidos dos diferentes cortes comerciais, 
Tabela 1 - Equações de predição de cortes cárneos de carcaças de suínos, por meio da área de olho de lombo, por ultra-som em tempo real (AOLUS)

Table 1 - Prediction equations of meat cuts swines carcass, by means of loin eye area, by ultrasound in real time

\begin{tabular}{|c|c|c|c|c|c|}
\hline $\begin{array}{l}\text { Componente } \\
\text { Component }\end{array}$ & $\begin{array}{l}\text { Regressão } \\
\text { Regression }\end{array}$ & $\mathrm{F}$ & Sig. & $\mathrm{R} 2$ & DPR SRD \\
\hline $\begin{array}{l}\text { Peso do pernil } \\
\text { Ham weight }\end{array}$ & $\hat{\mathrm{Y}}=-2,3201+0,4916$ AOLUS & 100,18 & 0,0001 & 0,94 & 1,23 \\
\hline $\begin{array}{l}\text { Peso da paleta } \\
\text { Shoulder weight }\end{array}$ & $\hat{Y}=-2,0443+0,4684$ AOLUS & 156,71 & 0,0000 & 0,96 & 0,94 \\
\hline $\begin{array}{l}\text { Peso do carré } \\
\text { Carré weight }\end{array}$ & $\hat{\mathrm{Y}}=-4,6082+0,4881$ AOLUS & 121,61 & 0,0000 & 0,95 & 1,11 \\
\hline $\begin{array}{l}\text { Peso da copa } \\
\text { Loin copa weight }\end{array}$ & $\hat{\mathrm{Y}}=-0,1725+0,1162$ AOLUS & 76,31 & 0,0001 & 0,93 & 0,33 \\
\hline $\begin{array}{l}\text { Peso da barriga } \\
\text { Bellyweight }\end{array}$ & $\hat{Y}=-3,2961+0,4219$ AOLUS & 55,74 & 0,0003 & 0,90 & 1,41 \\
\hline
\end{tabular}

AOLUS = Área de olho de lombo por ultra-som.

AOLUS = Loin eye area by ultra-sound.

optou-se por separar os dados de pernil, paleta, carré, copa e barriga. Para facilitar a visualização, estes dados estão apresentados na Tabela 2 , constatando-se que: 1) todas as equações para predição de músculo, gordura e ossos apresentaram $\mathrm{R}^{2}$ superior a 0,90 e DPR inferior a 1,00 , exceto para o corte barriga, em que o $\mathrm{R}^{2}$ para músculo foi de 0,84 , para o corte copa, no qual o $\mathrm{R}^{2}$ para gordura foi de 0,87 , e, também, para os cortes copa e barriga, nos quais o $\mathrm{R}^{2}$ para ossos foi de 0,87 e 0,83 , respectivamente e 2 ) todas as equações para predição de pele apresentaram $\mathrm{R}^{2}$ baixos, inferiores a 0,80 , exceto no corte carré.

O corte da barriga foi o que apresentou a maior dificuldade em ser dissecado, devido à grande quantidade de gordura entremeada com a carne, na região compreendida pelo bacon, porém, por meio dos dados mostrados na Tabela 2, é possível verificar que o $\mathrm{R}^{2}$ para estimar o músculo da barriga, apesar de ser mais baixo que os apresentados nos demais cortes, ainda está acima daqueles observados por outros autores (GRESHAM et al., 1994; LIU e STOUFFER, 1995; e BRONDUM et al., 1998).

Assim, verifica-se que, com exceção de pele, os demais tecidos podem ser preditos por equações a partir da informação área de olho de lombo nos suínos in vivo, por ultra-sonografia em tempo real.

As equações de predição de tecidos nos cortes cárneos de carcaças de suínos e de espessura de toucinho e da área de olho de lombo, por meio da área de olho de lombo medida por ultra-sonografia em tempo real, estão apresentadas na Tabela 3 , na qual se pode verificar que, novamente, com relação ao tecido pele, as demais equações apresentaram-se com $\mathrm{R}^{2}$ superiores a 0,90 .

Quanto às estimativas para medir a quantidade de tecidos, músculo, gordura, ossos e pele nas carcaças de suínos, encontraram-se valores de $\mathrm{R}^{2}$ bastante satisfatórios, pois para quantidade de músculo o R2 foi de 0,93 , com desvio-padrão residual de 3,34, ao passo que os resultados de GRESHAM et al. (1994) foram de 0,60 e 3,70 , respectivamente. Os resultados de gordura foram mais expressivos, com $\mathrm{R}^{2}$ de 0,97 e desvio-padrão residual de 1,05 , contra os resultados de GRESHAM et al. (1994), que foram de 0,57 e 2,75, respectivamente.

Por outro lado, LIUESTOUFFER(1995), utilizando dados de 325 animais, abatidos ao peso de mercado, avaliados com imagens ultra-sônicas em tempo real, com medições longitudinais, encontraram estimativas para peso de músculo livre de gordura e osso, com $\mathrm{R}^{2}$ de 0,88 e desvio-padrão residual de 1,19 .

Segundo alguns autores, o limite máximo de desvio-padrão residual para estimativa de equações para predição de carne em carcaças de suínos é de 2,50 (DIESTRE et al., 1989; HULSEGGE et al., 1994), porém, convém salientar que estes estudos foram desenvolvidos na Comunidade Européia, utilizando como preditores a pistola óptica Hennesy, em que o $\mathrm{R}^{2}$ raramente atinge 0,90 .

Outro fato a ser considerado é o número de animais avaliados, pois GRESHAM et al. (1994) utilizaram 96 animais abatidos, enquanto LIU e STOUFFER (1995), 325 animais.

Contudo, os resultados obtidos nesta pesquisa para estimar quantidade de músculos foram muito semelhantes aos encontrados por WAGNER et al. (1999), que, trabalhando com equações lineares, obtiveram $R^{2}$ de 0,94 e desvio-padrão residual de 3,15.

Os dados para estimar a espessura de toucinho e a área de olho de lombo, apresentados também na Tabela 3, 
Rev. bras. zootec.

Tabela 2 - Equações de predição da composição em tecidos do corte cárneo pernil em carcaças de suínos, determinadas via área de olho de lombo por ultra-sonografia em tempo real

Table 2 - Prediction equations of ham meat cuts tissues composition in swines carcass, determined by loin eye area, by ultrasound in real time

\begin{tabular}{|c|c|c|c|c|c|}
\hline $\begin{array}{l}\text { Componente } \\
\text { Component }\end{array}$ & $\begin{array}{l}\text { Regressão } \\
\text { Regression }\end{array}$ & $\mathrm{F}$ & Sig. & $\mathrm{R}^{2}$ & DPR SRD \\
\hline $\begin{array}{l}\text { Músculo do pernil } \\
\text { Ham muscle }\end{array}$ & $\hat{\mathrm{Y}}=-1,9289+0,3406$ AOLUS & 95,86 & 0,0001 & 0,94 & 0,87 \\
\hline $\begin{array}{l}\text { Gordura do pernil } \\
\text { Ham fat }\end{array}$ & $\hat{Y}=-0,9042+0,0926$ AOLUS & 162,83 & 0,0000 & 0,96 & 0,44 \\
\hline $\begin{array}{l}\text { Ossos do pernil } \\
\text { Ham bones }\end{array}$ & $\hat{\mathrm{Y}}=-0,1757+0,0445$ AOLUS & 78,13 & 0,0001 & 0,93 & 0,13 \\
\hline $\begin{array}{l}\text { Pele do pernil } \\
\text { Ham skin }\end{array}$ & $\hat{Y}=-0,3950+0,0108$ AOLUS & 13,82 & 0,0099 & 0,70 & 0,07 \\
\hline $\begin{array}{l}\text { Músculo da paleta } \\
\text { Shoulder muscle }\end{array}$ & $\hat{\mathrm{Y}}=-1,0930+0,2440$ AOLUS & 97,91 & 0,0001 & 0,94 & 0,62 \\
\hline $\begin{array}{l}\text { Gordura da paleta } \\
\text { Shoulder fat }\end{array}$ & $\hat{Y}=-1,4687+0,1631$ AOLUS & 349,42 & 0,0000 & 0,98 & 0,22 \\
\hline $\begin{array}{l}\text { Ossos da paleta } \\
\text { Shoulder bones }\end{array}$ & $\hat{\mathrm{Y}}=-0,0316+0,0376$ AOLUS & 54,57 & 0,0003 & 0,90 & 0,13 \\
\hline $\begin{array}{l}\text { Pele da paleta } \\
\text { Shoulder skin }\end{array}$ & $\hat{Y}=-0,7674+0,0090$ AOLUS & 5,50 & 0,0575 & 0,48 & 0,10 \\
\hline $\begin{array}{l}\text { Músculo do carré } \\
\text { Carré muscle }\end{array}$ & $\hat{Y}=-2,2889+0,2763$ AOLUS & 81,73 & 0,0001 & 0,93 & 0,76 \\
\hline $\begin{array}{l}\text { Gordura do carré } \\
\text { Carré fat }\end{array}$ & $\hat{\mathrm{Y}}=-1,9131+0,1350$ AOLUS & 152,48 & 0,0000 & 0,96 & 0,27 \\
\hline $\begin{array}{l}\text { Ossos do carré } \\
\text { Carré bones }\end{array}$ & $\hat{Y}=-0,1492+0,0529$ AOLUS & 99,84 & 0,0001 & 0,94 & 0,13 \\
\hline $\begin{array}{l}\text { Pele do carré } \\
\text { Carré skin }\end{array}$ & $\hat{\mathrm{Y}}=0,0019+0,0155$ AOLUS & 143,57 & 0,0000 & 0,96 & 0,03 \\
\hline $\begin{array}{l}\text { Músculo da copa } \\
\text { Loin copa muscle }\end{array}$ & $\hat{\mathrm{Y}}=-0,0978+0,0818$ AOLUS & 83,99 & 0,0001 & 0,93 & 0,22 \\
\hline $\begin{array}{l}\text { Gordura da copa } \\
\text { Loin copa fat }\end{array}$ & $\hat{Y}=-0,0339+0,0139$ AOLUS & 39,51 & 0,0008 & 0,87 & 0,06 \\
\hline $\begin{array}{l}\text { Ossos da copa } \\
\text { Loin copa bones }\end{array}$ & $\hat{\mathrm{Y}}=0,1661+0,0142$ AOLUS & 40,88 & 0,0007 & 0,87 & 0,06 \\
\hline $\begin{array}{l}\text { Músculo da barriga } \\
\text { Belly muscle }\end{array}$ & $\hat{Y}=-1,5363+0,2149$ AOLUS & 32,62 & 0,0012 & 0,84 & 0,94 \\
\hline $\begin{array}{l}\text { Gordura da barriga } \\
\text { Belly fat }\end{array}$ & $\hat{Y}=-2,2493+0,1647$ AOLUS & 81,22 & 0,0001 & 0,93 & 0,46 \\
\hline $\begin{array}{l}\text { Ossos da barriga } \\
\text { Belly bone }\end{array}$ & $\hat{\mathrm{Y}}=0,1807+0,0251$ AOLUS & 30,27 & 0,0015 & 0,83 & 0,11 \\
\hline $\begin{array}{l}\text { Pele da barriga } \\
\text { Belly skin }\end{array}$ & $\hat{\mathrm{Y}}=5,7516-0,0856$ AOLUS & 6,01 & 0,0496 & 0,50 & 0,33 \\
\hline
\end{tabular}

AOLUS = Área de olho de lombo por ultra-som.

AOLUS $=$ Loin eye area by ultra-sound.

demonstram mais uma vez $\mathrm{R}^{2}$ alto para espessura de toucinho e área de olho de lombo, com desvio-padrão residual de 0,85 e 3,08, respectivamente.

Em um trabalho utilizando o ultra-som na linha de abate, BRONDUM et al. (1998) avaliaram 450 carcaças e obtiveram valores de $\mathrm{R} 2$ de 0,88 e 0,77 , para espessura de toucinho e área de olho de lombo, e valores de desviopadrão residual de 1,24 e 2,90, respectivamente. Dessa forma, os dados apresentados na Tabela 3 estão de acordo com os resultados obtidos por vários pesquisadores, mesmo utilizando outras técnicas para predição.
Constam na Tabela 4 as correlações simples existentes entre os principais cortes comerciais da carcaça, como o corte do pernil, o músculo do pernil, músculo da paleta, o músculo da copa e a porcentagem de músculo na carcaça total, juntamente com as características de área de olho de lombo e espessura de toucinho, medidas na carcaça, e a área de olho de lombo, medida com ultra-som, em tempo real.

Verificou-se, também, que a espessura de toucinho, medida por ultra-som, na altura da décima costela, apresentou correlação de 0,95 com a espes- 
DUTRA JR et al.

Tabela 3 - Equações de predição de tecidos nos cortes cárneos de carcaças de suínos e de espessura de toucinho e área de olho de lombo, por meio da área de olho de lombo medida por ultra-sonografia em tempo real

Table 3 - Prediction equations of meat cuts tissues composition in swines carcass and backfat thickness and loin eye area, determined by loin eye area measured by ultrasound in real time

\begin{tabular}{|c|c|c|c|c|c|}
\hline $\begin{array}{l}\text { Componente } \\
\text { Component }\end{array}$ & $\begin{array}{l}\text { Regressão } \\
\text { Regression }\end{array}$ & $\mathrm{F}$ & Sig. & $\mathrm{R}^{2}$ & DPR SRD \\
\hline Gordura total & $\hat{\mathrm{Y}}=-6,9349+1,1576$ AOLUS (LEAUS) & 75,14 & 0,0001 & 0,93 & 3,34 \\
\hline $\begin{array}{l}\text { Total fat } \\
\text { Músculo total } \\
\text { Total muscle }\end{array}$ & $\hat{\mathrm{Y}}=-6,5692+0,5693$ AOLUS (LEAUS) & 184,62 & 0,0000 & 0,97 & 1,05 \\
\hline $\begin{array}{l}\text { Ossos total } \\
\text { Total bones }\end{array}$ & $\hat{Y}=-2,2252+0,3136$ AOLUS (LEAUS) & 89,12 & 0,0001 & 0,94 & 0,83 \\
\hline $\begin{array}{l}\text { Pele total } \\
\text { Total skin } \\
\text { ET }\end{array}$ & $\hat{\mathrm{Y}}=1,1402+0,0528$ AOLUS (LEAUS) & 27,43 & 0,0019 & 0,82 & 0,25 \\
\hline $\begin{array}{l}B T \\
\mathrm{AOL}\end{array}$ & $\hat{\mathrm{Y}}=0,4884+0,2946$ AOLUS (LEAUS) & 74,44 & 0,0001 & 0,93 & 0,85 \\
\hline LEA & $\hat{\mathrm{Y}}=6,1258+0,9235$ AOLUS (LEAUS) & 56,17 & 0,0003 & 0,90 & 3,08 \\
\hline
\end{tabular}

AOLUS = Área de olho de lombo por ultra-som.

$\mathrm{ET}=\quad$ Espessura de toucinho.

$\mathrm{AOL}=$ Área de olho de lombo.

LEAUS $=$ Loin eye area by ultra-sound.

$B T=\quad$ Backfat thickness.

$\angle E A=\quad$ Loin eye area.

Tabela 4 - Correlações entre AOL medidas por ultra-som com características de carcaças obtidas por dissecação Table 4 - Correlation among ELA measured by ultrasound and carcass traits obtained by dissection

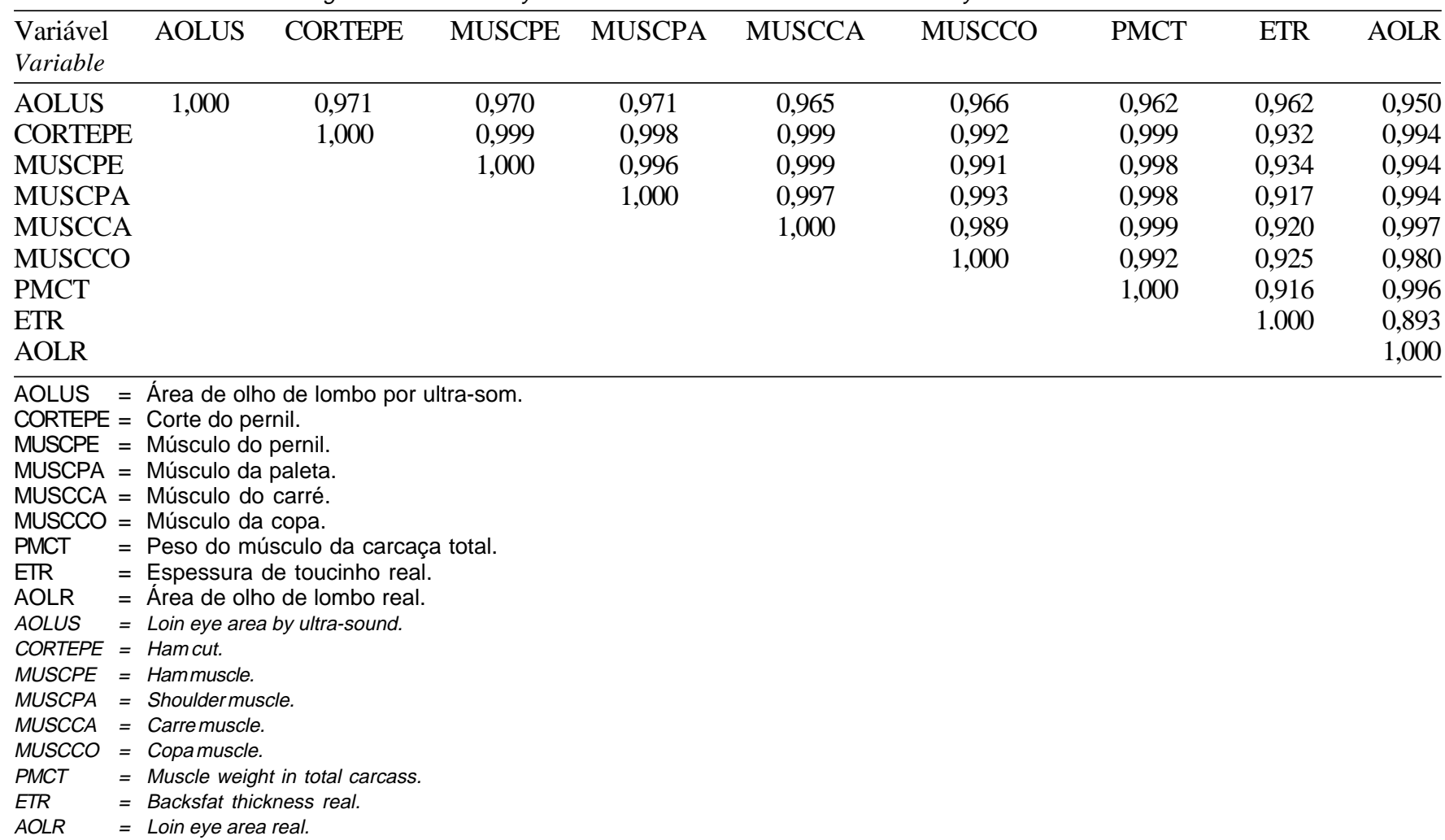


Rev. bras. zootec.

sura de toucinho medida na carcaça. Estes resultados são superiores aos encontrados por BUSK (1986), que utilizou um equipamento Aloka SSD-210DX, obtendo coeficientes de correlações de 0,87 e 0,90 para medidas de espessura de toucinho na décima e última costela, respectivamente. Já HOUGHTON e TURLINGTON (1992) encontraram correlações de 0,93. Porém, SCHINCKEL et al. (1994), trabalhando com um equipamento Aloka SSD-500, obtiveram índices de correlações de 0,92 e 0,94 para as medidas de ET, tomadas na décima e última costela, respectivamente.

\section{Conclusões}

A técnica de ultra-sonografia em tempo real apresentou alta correlação com as medidas tomadas nas carcaças dos suínos, podendo ser utilizada para estimar a quantidade de carne na carcaça e, também, o rendimento dos cortes comerciais com precisão.

\section{Referências Bibliográficas}

BRONDUM, J., EGEBO, M., AGERSKOV, C., BUSK, H. 1998. On-line pork carcass grading with the Autofom ultrasound system, J. Anim. Sci., 76(7):1859-1868.

BUSK, H. 1986. Testing of 5 ultrasonic equipment's for measuring of carcass quality on live pigs. World Review Anim. Prod., 22(3):35-38.

DIESTRE, A., GISPERT, M., OLIVER, M.A. 1989. The evaluation of automatic probes in Spain for the new scheme for pig carcass grading according to the $\mathrm{E} \mathrm{C}$ regulations. Anim. Prod., 48(2):443-448.

GRESHAM, J.D., McPEAKE, S.R., BERNARD, J.K. et al. 1994. Prediction of live and carcass characteristics of market hogs by use of single longitudinal ultrasonic scan. J. Anim. Sci., 76(6):1409-1416.

HOUGHTON, P.L., TURLINGTON, L.M. 1992. Application of ultrasound for feeding an finishing animals: a review. J. Anim. Sci., 70(3):930-941.

HULSEGGE, B., STERRENBURG, P., MERKUS, G.S.M. 1994. Prediction of lean meat proportion in pig carcasses and in the major cuts from multiple measurements made with the Hennessey Grading Probe. Anim. Prod., 59(1):119-123.

IRGANG, R., GUIDONI, A.L., BERLITZ, D. et al. 1998.
Medidas de espessura de toucinho e de profundidade de músculo para estimar rendimento de carne magra em carcaças de suínos, R. Bras. Zootec., 27(5):928-935.

JOYAL, S.M., JONES, S.D.M., KENNEDY, B.W. 1987. Evaluation of electronic Meat-Measuring Equipment in predicting carcass composition in the live pig. Anim. Prod., 45(1):97-102.

LIU, Y. 1997. AUSKEY FOR WINDOWS VERSION 5.00, ANIMAL ULTRASOUND SERVICES INC, 95 Brown Road Room 248, Ithaca NY, 14850, Users Manual, Copyright. 192p.

LIU, Y., STOUFFER, J.R. 1995. Pork carcass evaluation with on automated and computerized ultrasonic system. J. Anim. Sci., 73(1):29-38.

LO, L.L., McLAREN, D.G., McKEITH, F.K. et al. 1992a Genetic analyses of growth real-time ultrasound carcass and pork quality traits in Duroc and Landrace pigs: I Breed effects. J. Anim. Sci., 70(8):2373-2386.

LO, L.L., McLAREN, D.G., McKEITH, F.K. et al. 1992b. Genetic analyses of growth real-time ultrasound carcass and pork quality traits in Duroc and Landrace pigs: II Heritabilities and correlation's. J. Anim. Sci., 70(8):2387-2396.

SATHER, A.P., BAILEY, D.R.C., JONES, S.D.M. 1996. Realtime ultrasound image analysis for the estimation of carcass yield an pork quality. Can. J. Anim. Sci., 76(1):55-62.

TERRY, C.A. SAVELL, J.W., RECIO, H.A., CROSS, H.R. 1989. Using ultrasound technology to predict pork carcass composition. J. Anim. Sci., 67(5):1279-1284.

THWAITES, C.J. 1984. Ultrasonic estimation of carcasses composition. Australian Meat Research Committee, 47.

UNIVERSIDADE FEDERAL DE VIÇOSA - UFV. 1998. SAEG - Sistema de análises estatísticas e genéticas. Versão 7.1. Viçosa, MG. 150p. (Manual do usuário).

WAGNER, J.R., SCHINCKEL, A.P., CHEN, W. et al. 1999. Analysis of body composition changes of swine during growth and development. J. Anim. Sci., 77:1442-1466.

WILSON, D.E. Improvement beef cattle genetics using ultrasound. In: CONGRESSO BRASILEIRO DE ZOOTECNIA, 6, PUCRS, Porto Alegre, 1996. Anais... Porto Alegre, 1996. p.169-200.

Recebido em: 27/09/00

Aceito em: 15/05/01 